\title{
A thrombus in the left anterior descending artery after the use of multiple alternative medicines
}

\author{
Sachin Kumar Amruthlal Jain*, Brijesh Patel, Timothy Larsen, Patrick Alexander and Shukri \\ David
}

Department of Internal Medicine, Section of Cardiology, Providence Hospital and Medical Centers, 16001 West Nine Mile Road, Southfield, MI 48075, USA.

Accepted 21 December, 2012

\begin{abstract}
In the United States many patients turn to alternative medications. These substances are neither tested nor approved by U.S. Food and Drug Administration. Knowledge of their side effect profile is lacking. Several of these substances are biologically active at very low concentrations and can therefore exert powerful physiologic effects. Lachesis mutus and Crotalus horridus are snake venoms that can damage vessel intima and promote platelet aggregation. By altering several aspects of Virchow's classic triad, these substances can potentially result in severe adverse events, including myocardial infarction and venous thrombosis. Herein, we report a case of a young woman who presented to the emergency room at our hospital complaining of acute onset chest pain. Detailed history revealed that the patient ingested various substances without her knowledge of side effect profile. Further diagnostic work up revealed an acute ST-Segment Elevation Myocardial Infarction due to a thrombus in the left anterior descending artery. Although, there is a plenty of data available through laboratory research describing the properties of some of the substances described in the case; almost none are integrated in patient care. Through this case report, we would like to increase the awareness among practicing health care providers regarding various substances patients may be using without consideration of potential consequences.
\end{abstract}

Key words: Myocardial infarction, alternative medicine, Lachesis mutus, Crotalus horridus, thrombosis, snake venom.

\section{INTRODUCTION}

Certain forms of alternative medicine have been in practice for several millennia. By definition, alternative medicine is something that differs from or is outside the scope of conventional medical practice (Bratma, 1997). In an effort to seek relief, many patients turn to alternative therapies. Patients who use these substances generally assume they are safe as these substances are purchased over the counter, contain "natural" ingredients, and are recommended by alternative medical practitioners.

${ }^{*}$ Corresponding author. E-mail: doctorsachin@gmail.com. Tel: 248-849-3151. Fax: 248-849-3230.
The vast majority of these substances are neither tested nor approved by the US Food and Drug Administration (FDA). These substances are not classified as "drugs" by FDA and therefore are not subject to rigorous safety testing. Patients and physicians are generally not aware of the potential risks that these remedies may carry.

\section{CASE REPORT}

A 34 year old female presented to the emergency room complaining of sudden onset severe stabbing substernal chest pain that radiated to her back. The pain started while walking. The initial episode lasted $20 \mathrm{~min}$ and resolved with rest. The pain returned with minimal exertion. She was moderately short of breath but 


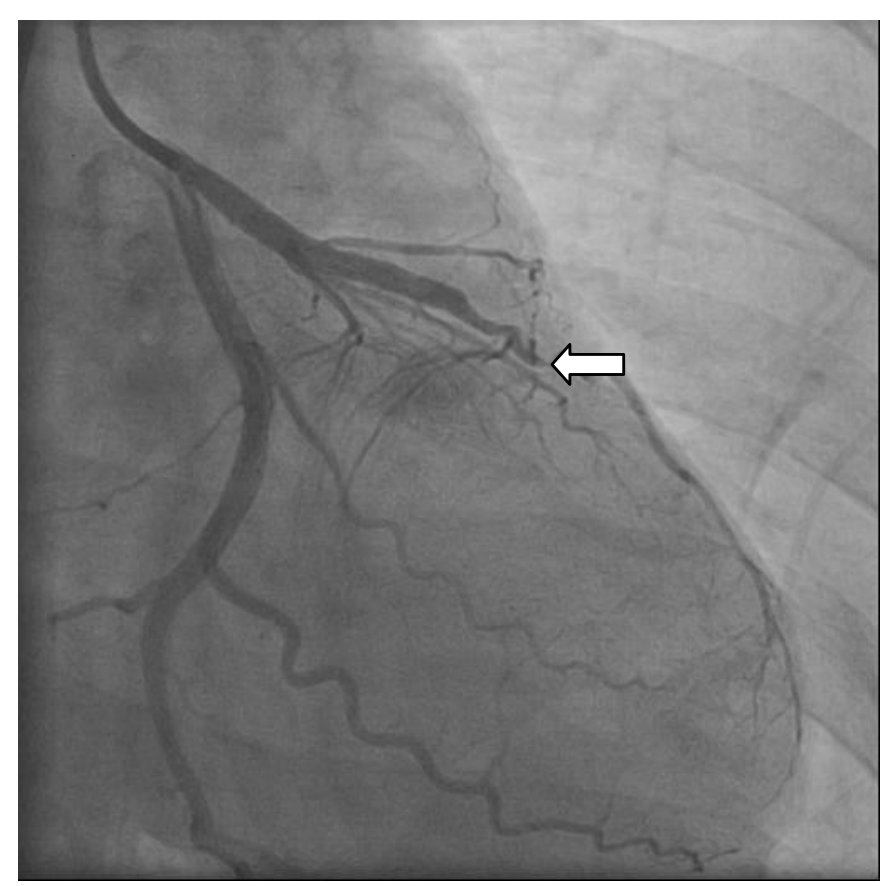

Figure 1. Complete occlusion of the mid-left anterior descending coronary artery by a thrombus (White arrow).

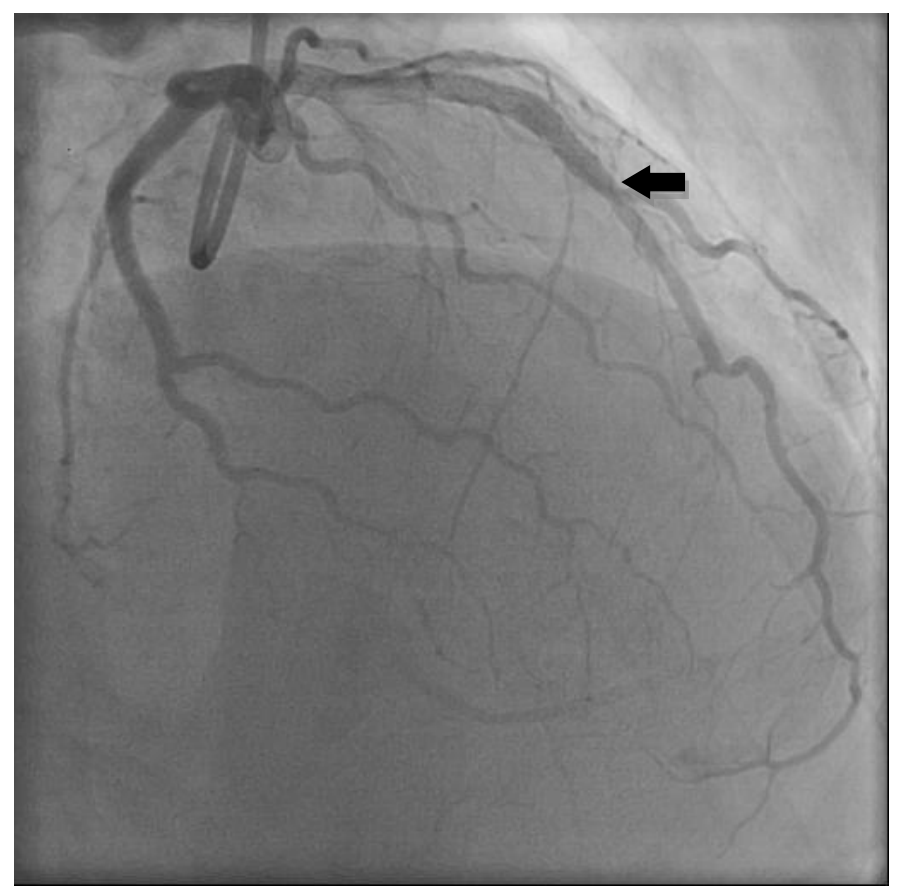

Figure 2. After thrombectomynand stenting of the mid-left anterior descending coronary artery demonstrating restoration of flow (Black arrow).

denied nausea, light-headedness, palpitations, or diaphoresis.

Her medical history was significant for gluten intolerance and exercise induced asthma; there was no history of diabetes, hyper- tension, dyslipidemia, or tobacco abuse. There was no family history of heart disease or sudden cardiac death.

She denied taking prescription medications, she had, however, been taking multiple alternative medications in hopes of curing her gluten sensitivity. These included a diet pill, Lachesis mutus $12 \mathrm{X}$ (pit viper venom), Bella Donna 12X, Crotalus horridus 12X (rattle snake venom), lyssin (saliva of rabid dog), pyrogenium 12X (made from beef left standing in water for 3 weeks), and Tarantula cubensis $12 \mathrm{X}$ (dehydrated, ground tarantula).

Initial vitals were: temperature $\left(98.5^{\circ} \mathrm{F}\right)$, blood pressure $(100 / 58$ $\mathrm{mmHg}$ ), heart rate (83 beats/min), respiratory rate (18 breaths $/ \mathrm{min})$, and oxygen saturation (99\%) on room air. There were no carotid, abdominal, or femoral bruits. On chest auscultation, the heart rate was regular, there were no murmurs or gallops, lungs were clear without adventitious lung sounds, the point of maximal impulse was not displaced. Peripheral pulses were strong and equal in the bilateral carotid, radial, femoral, dorsalis pedis, and posterior tibial arteries. The remaining examination was unremarkable.

Initial troponin was $0.02 \mathrm{ng} / \mathrm{ml}$ (Normal values: 0.00 to 0.10 $\mathrm{ng} / \mathrm{ml}$ ); repeated $6 \mathrm{~h}$ apart were 0.31 and $0.37 \mathrm{ng} / \mathrm{ml}$; complete blood count, metabolic panel, and renal, liver, and thyroid function tests were normal. There were no ischemic changes on serial electrocardiograms (EKGs). She was referred for cardiac catheterization which identified an acute thrombus in the mi-left anterior descending artery (Figure 1). The patient's right coronary artery was patent. Thrombectomy was performed and a stent was placed (Figure 2). After the procedure, she was free of pain without residual coronary artery stenosis.

Subsequent evaluation for the presence of a hypercoaguable condition was unrevealing. Protein $\mathrm{C}$ and $\mathrm{S}$ levels, antithrombin III activity, serum homocysteine, antinuclear antibody, and antiphospholipid antibody tests were normal. There was no mutation in the prothrombin gene.

\section{DISCUSSION}

Many physicians feel that while evidence supporting the use of alternative medications is lacking, overall these substances pose a low risk with the worst case scenario limited to obtaining a placebo effect. In a study of examining the effectiveness of several alternative medications, Shang et al. (2005) concluded that many do not perform better than the placebo. These substances, however, are not entirely benign.

This case illustrates the potentials risks associated with ingesting unknown and untested medications. These substances have not been studied in order to determine safety and efficacy. Some of the snake venoms are known to alter hemostasis (Leeser, 1958). The Lachesis mutus and Crotalus horridus venoms are believed to cause damage to the vessel intima and promote platelet aggregation (Leeser, 1958). Even though most substances are significantly diluted prior to administration, several are active at very low concentrations. Eagle (1937) demonstrated that snake venom is still active up to a 1:4000000 dilution.

The Naranjo probability scale identifies our patient's myocardial infarction as a probable adverse drug reaction (Naranjo et al., 1985). We suspect $C$. horridus and/or $L$. mutus to be the culprits; however, given the lack of information regarding physiologic effects, any of the other substances may be directly or indirectly involved. 


\section{Conclusion}

The case presented underscores the importance of detailed history taking including up to date lists of over the counter and alternative medications. Clinicians and patients must consider the potential harms of these unproven and unregulated remedies. For majority of these substances, the pharmacological mechanisms, drug interactions, and side effect profiles are unknown. A prescriber must take these factors into consideration before recommending an alternative medication. Patients must be educated on potential risks associated with these remedies.

\section{REFERENCES}

Bratma S (1997). The Alternative Medicine Sourcebook. Lowell House. p. 7.

Eagle $H$ (1937). The coagulation of blood by snake venoms and its physiologic significance. J. Exp. Med. 65:613.
Leeser O (1958). Actions and medicinal use of snake-venoms. Br. Homeopath.J. 47:153.

Naranjo CA, Busto U, Sellers EM, Sandor P, Ruiz I, Roberts EA, Janecek E, Domecq C, Greenblatt DJ (1981). A method for estimating the probability of adverse drug reactions. Clin. Pharmacol. Ther. 30(2):239-245.

Shang A, Huwiler-Müntener K, Nartey L, Jüni $P$, Dörig $S$, Sterne JA, Pewsner D, Egger M (2005). Are the clinical effects of homeopathy placebo effects? Comparative study of placebo-controlled trials of homoeopathy and allopathy. Lancet 366(9487):726-732. 